\title{
Waldorf- und Regelschulen in Österreich
}

\author{
Kompetenzvergleiche mit Detailanalysen zur Lesekompetenz- \\ PISA 2018
}

Christina Wallner-Paschon ${ }^{1}$, Lisa Wiesinger ${ }^{2}$

https://doi.org/10.53349/resource.2021.i16.a994

\section{Zusammenfassung}

Mit PISA werden alle drei Jahre die Kompetenzen der Jugendlichen in Lesen, Mathematik und Naturwissenschaft erfasst. In Österreich beteiligen sich seit PISA 2000 auch die Waldorfschulen an diesen Erhebungen. Der Beitrag widmet sich, nach einer kurzen Charakterisierung der Waldorfschüler*innen hinsichtlich Geschlechterverteilung sowie Berufsstatus und Bildungsniveau der Eltern, den Ergebnissen in Lesen, Mathematik und Naturwissenschaft. Dabei werden mit Hilfe von Leistungsmittelwerten und Kompetenzstufen die Schüler*innen aus Waldorfschulen jenen aus den österreichischen Regelschulen gegenübergestellt. Die Analysen zur Leistungsentwicklung der Waldorfschüler*innen zeigen über die PISA-Erhebungsjahre relativ stabile Ergebnisse in allen drei Kompetenzbereichen. Während sich Schüler*innen in Waldorf- und Regelschulen in ihren Kompetenzen unterscheiden, sind die Geschlechterunterschiede in den drei Kompetenzbereichen bei Schüler*innen von Waldorfschulen ähnlich hoch ausgeprägt wie bei Schüler*innen von Regelschulen. Detailanalysen zur Lesekompetenz zeigen, dass Waldorfschüler*innen im Vergleich zu den Jugendlichen in den Regelschulen ein deutliches Kompetenzprofil aufweisen. Sowohl die Lesefreude als auch das Leseselbstkonzept ist bei den Waldorfschüler*innen sehr positiv ausgeprägt.

$\begin{array}{ll}\text { Schlüsselwörter: } & \text { Keywords: } \\ \text { Bildungsforschung } & \text { Educational research } \\ \text { Waldorfschulen } & \text { Waldorf Schools } \\ \text { Alternativschulen } & \text { Alternative education } \\ \text { PISA } & \text { PISA }\end{array}$

\section{Einleitung}

Die Waldorfschulen als einzige größere Gruppe von Alternativschulen in Österreich unterscheiden sich in schulorganisatorischen, pädagogischen und didaktischen Merkmalen von den Regelschulen (im Überblick siehe Loebell, 2018, S. 246-251). Deshalb haben die Ergebnisse aus dem Vergleich mit dem österreichischen Regelsystem eine besondere Bedeutung. Wie bereits in vorangegangenen PISA-Erhebungen wurden auch 2018 die 15-/16-Jährigen in den österreichischen Waldorfschulen gemäß den bei PISA standardisierten Erhebungsverfahren getestet. Es handelt sich dabei um eine nationale Zusatzerhebung, an der zehn österreichische Waldorfschulen teilnahmen. Waldorfschulen werden auf Initiative von Eltern und Lehrpersonen gegründet und von den Lehrpersonen ohne die Instanz eines Direktoriums selbst verwaltet. Waldorfschulen sind Privatschulen mit Öffentlichkeitsrecht und verlangen Schulgeld, was mitunter ein Grund dafür ist, dass Waldorfschüler*innen vorwiegend aus der Mittelschicht bzw. dem Bildungsbürgertum stammen. Es handelt sich um Einheits- bzw. Gesamtschulen von der 1. bis zur 12. Schulstufe, wobei eine Klasse von der 1 . bis zur 8. Schulstufe im Normalfall von derselben Lehrperson unterrichtet wird. Waldorfschulen sind Ganztagsschulen mit verschränktem Unterricht, wodurch sich auch andere Möglichkeiten zur Förderung und Hilfestellung bieten. In

\footnotetext{
${ }^{1}$ Institut des Bundes für Qualitätssicherung im österreichischen Schulwesen (IQS), Alpenstraße 121, 5020 Salzburg; E-Mail: christina.wallner-paschon@iqs.gv.at

${ }^{2}$ Institut des Bundes für Qualitätssicherung im österreichischen Schulwesen (IQS), Alpenstraße 121, 5020 Salzburg
} 
der Regel wird beispielsweise ein differenzierter, kostenloser Förderunterricht oder Hausaufgabenhilfe angeboten (Verband der Waldorfschulen, 2005). Der Besuch einer Waldorfschule ist durch einen zensurfreien Bildungsweg bis zum Ende der 12. Klasse gekennzeichnet. Statt Zeugnisnoten - wie dies überwiegend in der österreichischen Regelschule der Fall ist - erhalten die Waldorfschüler*innen eine Verbalbeurteilung. Der Lehrstoff ist in Epochen gegliedert und wird weitgehend ohne Benützung von Schulbüchern vermittelt (Hellmich, 1999). Mit dem Epochenunterricht bricht die Waldorfschule mit der Gewohnheit des Regelschulwesens, nach jeweils 50 Minuten in ein anderes Fach zu wechseln. Epochaler Unterricht erfolgt, indem täglich etwa zwei Stunden lang über einen Zeitraum von ca. vier Wochen ein und derselbe Themen- oder Fachbereich (z. B. Mathematik, Muttersprache, Sachunterricht) zum Unterrichtsinhalt wird (Rauthe, 1992).

Bis 2015 erfolgte die Erhebung, indem die Schüler*innen die Aufgaben in einem gedruckten Testheft bearbeiteten. Mit PISA 2015 wurde eine wesentliche Neuerung eingeführt: Seitdem erfolgt der Test mittels Computer. Details zu dieser und weiteren Änderungen (z. B. der Skalierungsmethodik) finden sich bei Suchań und Breit (2016, S. 34 f.). Durch diese Veränderungen ist der Punktevergleich zwischen PISA 2015 bis 2018 und früheren Erhebungen nur eingeschränkt interpretierbar. Die Daten ab PISA 2015 werden deshalb bei Trendvergleichen optisch abgegrenzt und nicht direkt mit vorangegangenen Erhebungen verglichen.

Darüber hinaus soll darauf hingewiesen werden, dass die PISA-Stichprobe relativ groß und präzise ist, weshalb auch sehr kleine Unterschiede zwischen Waldorf- und Regelschüler*innen statistisch signifikant werden können. Diese Unterschiede existieren zwar mit großer Wahrscheinlichkeit, sind aber manchmal so klein, dass sie in praktischer Hinsicht nicht relevant sind.

Bisherige Publikationen zu den österreichischen Waldorfschulen in Zusammenhang mit den PISA-Erhebungen finden sich bei Reiter (2002), Wallner-Paschon (2006, S. 143-154; 2009, S. 387-399, 2018) und Salchegger, Wallner-Paschon \& Bertsch (2021). Darüber hinaus sind empirische Forschungsergebnisse zur Waldorfpädagogik eher rar gesät. Die wenigen erfahrungswissenschaftlichen Untersuchungen in diesem Forschungsfeld beziehen sich vor allem auf retrospektive, subjektive Einschätzungen ehemaliger Schüler*innen oder/und weisen geringe Rücklauf- bzw. Teilnahmequoten auf (Barz \& Randoll, 2007; Randoll, 2004; Ullrich, 2004; Liebenwein, Barz \& Randoll, 2012). Im vorliegenden Beitrag werden die Daten aus PISA 2018 zu den kognitiven und nicht-kognitiven Kompetenzen von Waldorf- und Regelschüler*innen gegenübergestellt.

\section{Populationsbeschreibung}

Bei der nationalen Zusatzstichprobe handelt es sich im Gegensatz zur regulären PISA-Stichprobe um eine Vollerhebung der österreichischen 15-/16-jährigen Waldorfschüler*innen. An der Untersuchung beteiligen sich immer alle österreichischen Waldorfschulen mit Schüler*innen der Zielpopulation ${ }^{3}$. Bei PISA 2018 beträgt die Teilnahmerate rund 97 \% (167 Waldorfschüler*innen) und ist um 2 Prozentpunkte höher als jene der regulären PISA-Stichprobe mit rund 95 \% (Glaeser \& Pareiss, 2019). Obwohl es sich bei dieser nationalen Zusatzerhebung um eine Vollerhebung mit hohem Rücklauf handelt, muss darauf hingewiesen werden, dass Analyseergebnisse, die aus einer relativ kleinen Population gewonnen werden (rund 140-170 Schüler*innen aus 10 Waldorfschulen), starken Schwankungen über die Zeit unterliegen können. Leistungs- oder Merkmalsunterschiede zwischen den Erhebungszeitpunkten resultieren somit nicht zwangsläufig aus pädagogischen Veränderungen, sondern können durch natürliche Jahrgangsunterschiede entstehen.

Die Schüler*innen der Waldorfschulen unterscheiden sich von Jugendlichen des Regelschulwesens in einigen bedeutenden Merkmalen wie beispielsweise Geschlechterverteilung, Berufsstatus und Bildungsniveau der Eltern. Wie in allen bisherigen Erhebungen gibt es in Waldorfschulen mehr Mädchen als Burschen (WallnerPaschon, 2006, 2009, 2018). In PISA 2018 ist der Mädchenanteil um 6 Prozentpunkte höher als in den Regelschulen (bei PISA 2015 waren es 5 \%). Darüber hinaus haben fast 80 \% der Jugendlichen in Waldorfschulen zumindest einen Elternteil mit akademischer Bildung, während es in den Regelschulen (Österreich gesamt) etwa ein Drittel ist (36 \%). Über 90 \% der Jugendlichen in Waldorfschulen haben Eltern mit zumindest Matura als höchstem Abschluss; in Regelschulen sind es im Vergleich dazu 57 \%. Auf der anderen Seite stellen Jugendliche, deren Eltern eine Lehre bzw. eine berufsbildende mittlere Schule als höchsten Bildungsabschluss vorzuweisen haben, mit 35 \% eine relativ große Gruppe in Regelschulen dar, während diese Gruppe in Waldorfschulen mit $7 \%$ vergleichsweise klein ist (s. Abbildung 1 ).

\footnotetext{
${ }^{3}$ Die Grundgesamtheit bei PISA wird altersbasiert definiert und umfasst nur die beschulte Population der definierten Altersgruppe (Schüler*innen, die zum Testzeitpunkt zwischen 15 Jahre drei Monate und 16 Jahre zwei Monate alt sind und die mindestens die 7. Schulstufe besuchen) (Pareiss, 2018, S. 35).
} 

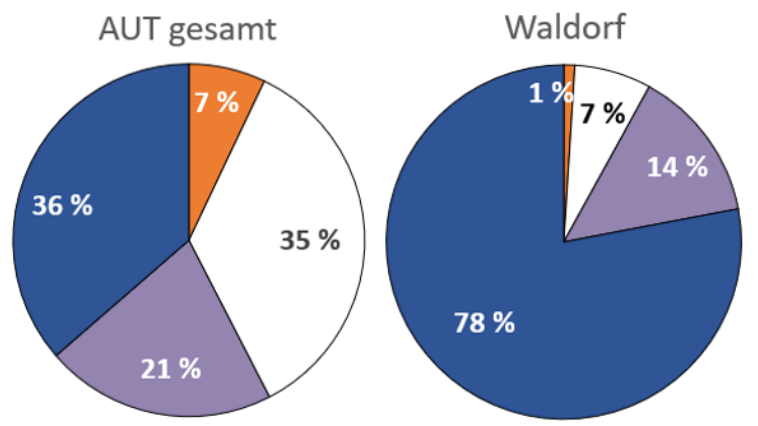

$\square$ maximal Pflichschulabschluss

$\square$ Lehre/BMS

$\square$ Matura

口Universität/FH

jeweils höherer Bildungsabschluss

der beiden Elternteile

Abbildung 1: Bildung der Eltern - Waldorf- und Regelschulen im Vergleich (PISA 2018).

Auch in Bezug auf den Berufsstatus der Eltern unterscheiden sich die Waldorf- von den Regelschulen. Der verwendete Index des sozioökonomischen Status, HISEI (Highest International Socio-Economic Index of occupational status), basiert auf dem höchsten beruflichen Status der Eltern (Ganzeboom et al., 1992). Der Wertebereich des HISEI-Index liegt zwischen 16 Punkten für ungelernte Hilfskräfte (z. B. Reinigungspersonal, landwirtschaftliche Hilfsarbeiter*innen) und 90 Punkten, was dem Berufsstatus von Richter*innen oder Ärzt*innen entspricht. Abbildung 2 zeigt die Verteilung des Berufsstatus (HISEI) getrennt für Eltern von Schüler*innen der regulären PISA-Population (Österreich gesamt) und der Waldorfschulen. Der HISEI der Eltern der Waldorfschüler*innen liegt mit durchschnittlich 69 Statuspunkten 18 Punkte über dem Durchschnittswert der Eltern von gleichaltrigen Schüler*innen des österreichischen Regelsystems. In Relation zu Österreich gesamt ist eine Häufung der Waldorfschüler*innen in den eher höheren Statusbereichen zu erkennen, während in den unteren Bereichen vergleichsweise wenig von ihnen anzutreffen sind.

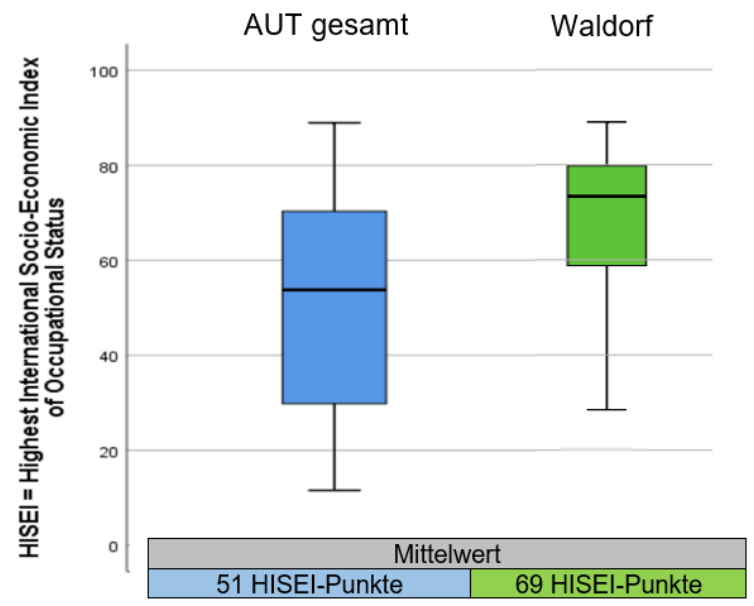

Abbildung 2: Berufsstatus der Eltern - Waldorf- und Regelschulen im Vergleich (PISA 2018).

Darüber hinaus unterscheidet sich die Schülerpopulation der Waldorfschulen auch hinsichtlich weiterer Merkmale, die in Zusammenhang mit den Schülerkompetenzen eine Rolle spielen. Aus anderen Untersuchungen ist bekannt, dass in Waldorfschulen der Anteil der Quereinsteiger*innen aus Regelschulen mit rund $37 \%$ relativ hoch ist (Liebenwein, Barz \& Randoll, 2012, S. 10). Weiters sind in Waldorfschulen Schüler*innen aus einer klassischen Kernfamilie deutlich unterrepräsentiert. Etwa $40 \%$ der Waldorfschüler*innen kommen aus Alleinerzieherhaushalten (ebd.). Die in diesem Abschnitt beschriebenen Besonderheiten der Waldorfpopulation im Vergleich zu den Jugendlichen der Regelschulen werden bei den hier berichteten Ergebnissen statistisch nicht kontrolliert und sind bei der Interpretation der Ergebnisse im Hintergrund zu berücksichtigen. 


\section{Kompetenzen der Waldorfschüler*innen im Vergleich}

In diesem Kapitel werden die Kompetenzen in Lesen, Mathematik und Naturwissenschaft von Jugendlichen in Regel- und Waldorfschulen über die PISA-Erhebungsjahre verglichen.

\subsection{Lesekompetenz}

Die Entwicklung der Lesekompetenz kann seit PISA 2000 im Trend beobachtet werden. Abbildung 3 zeigt die durchschnittliche Lesekompetenz der Waldorfschüler*innen im Vergleich zu jener der Schüler*innen in österreichischen Regelschulen für PISA 2000 bis 2012 sowie für 2015 bis 2018. Der österreichische Gesamtmittelwert ist durch graue Quadrate und der Mittelwert der Waldorfschulen durch rote Kreise gekennzeichnet.

Mit Ausnahme von PISA 2003 bewegen sich die Lesemittelwerte der Waldorfschüler*innen zwischen 492 und 501 Punkten und sind über die Zeit relativ stabil geblieben. Auch der aktuelle Lesemittelwert von 494 bei PISA 2018 liegt in dieser Hinsicht im Trend. Die Waldorfschüler*innen liegen bei PISA 2003 bis 2018 signifikant über dem Lese-Gesamtmittelwert von Österreich. Für PISA 2000 zeigen sich keine signifikanten Unterschiede in der Lesekompetenz von Jugendlichen in Waldorf- und Regelschulen.

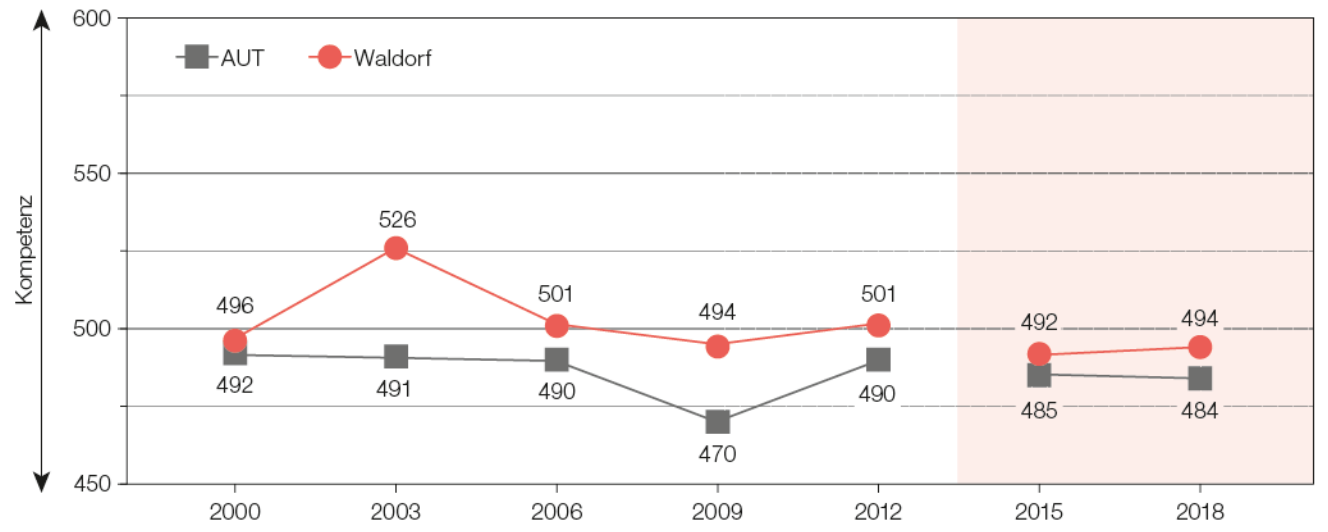

Abbildung 3: Veränderung der Durchschnittsleistung in Lesen - Waldorf- und Regelschulen im Vergleich (PISA 2000, 2003, 2006, 2009, 2012, 2015, 2018).

Neben der Analyse der Mittelwerte ist ein Vergleich der Verteilung dieser Leistung auf die Kompetenzstufen relevant. Bei PISA 2018 wird die Bandbreite der Aufgabenschwierigkeit in Lesen durch sieben Kompetenzlevels repräsentiert. Jede Aufgabe kann einer Kompetenzstufe zugeordnet werden. Dadurch ist es auch möglich, ergänzend zum Mittelwert die Leistungsverteilung der Jugendlichen näher zu beschreiben. Abbildung 4 stellt hierfür die prozentuellen Anteile der Waldorfschüler*innen in den sieben Kompetenzstufen für Lesen der österreichischen Gesamtstichprobe gegenüber.

Level $1 \mathrm{c}$ ist die unterste Kompetenzstufe, auf der die einfachsten PISA-Leseaufgaben verortet werden, gefolgt von Level $1 \mathrm{~b}$ und 1a, Level 2, Level 3 und so weiter - bis Level 6, der höchsten Kompetenzstufe. Schüler*innen, die weniger als 50 \% der einfachsten Aufgaben lösen können, fallen unter Level 1c. Als Risikogruppe gelten Schüler*innen auf den Kompetenzstufen 1a, 1b, 1c und darunter. Zur Spitzengruppe in Lesen zählen Schüler*innen auf den Kompetenzlevels 5 und 6 (im Detail siehe Suchań \& Höller, 2019, S. 31).

Auf den unteren Leistungslevels (Risikogruppe) befinden sich rund $16 \%(0,24 \%+2,97 \%+12,29 \%=$ 15,5 \%) der Waldorfschüler*innen. Den Leistungslevels 5-6 und damit der Spitzengruppe gehören $4 \%(3,86 \%+$ $0,00 \%=3,86 \%$ ) der Waldorfschüler*innen an. Im Vergleich zur Gesamtstichprobe der österreichischen Regelschulen weisen die Waldorfschulen eine um drei Prozentpunkte kleinere Spitzengruppe auf und liegen mit ihrem Risikogruppen-Anteil um rund 8 Prozentpunkte unter dem Österreich-Mittel. 


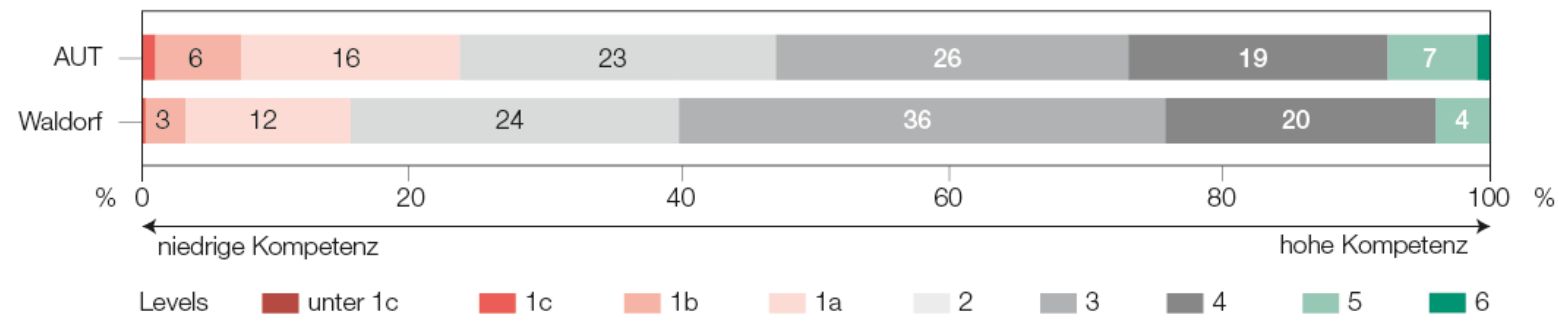

Abbildung 4: Kompetenzstufen in Lesen - Waldorf- und Regelschulen im Vergleich (PISA 2018).

Da die Lesekompetenz bei PISA 2018 schwerpunktmäßig erfasst wurde, ist eine detailliertere Leistungsanalyse möglich. Folgende drei Leseprozesse werden dabei genauer untersucht: (1) Informationen finden, (2) Verstehen und (3) Bewerten und Reflektieren (im Detail siehe Höller \& Toferer, 2019, S. 15 f). In Abbildung 5 sind für jeden dieser drei kognitiven Leseprozesse sowie für die Bearbeitung von Texten aus einer oder mehreren Quellen die Mittelwerte und Konfidenzintervalle für die österreichischen Regelschulen in Blau eingetragen. Die durchschnittliche Leistung der Waldorfschüler*innen ist jeweils mit einem grünen Kreis gekennzeichnet.

Österreichs Jugendliche zeigen bei den Leseprozessen sehr ähnliche Ergebnisse, das heißt, es fällt ihnen keiner dieser drei Prozesse leichter oder schwerer. Bei den Jugendlichen in Waldorfschulen zeigt sich im Gegensatz dazu ein klares Kompetenzprofil. Ihre relativen Stärken liegen bei den kognitiven Prozessen Verstehen und Bewerten von Texten - hier liegen sie mit mehr als 10 Testleistungspunkten über dem Durchschnittswert der Regelschüler*innen. Beim Auffinden von Informationen in Texten sind sie jedoch signifikant schlechter als Jugendliche in Regelschulen. Auch in Bezug auf die im Test eingesetzte Textstruktur gibt es für Österreich keine wesentlichen Leistungsunterschiede. Jugendliche in Waldorfschulen zeigen jedoch auch hier ein klares Profil. Ihre Stärke liegt beim Bearbeiten von Texten aus einer Quelle - hier liegen sie um 15 Testpunkte über den AUTSchnitt. Beim Bearbeiten von Texten aus multiplen Quellen liegen sie mit den Regelschüler*innen gleich auf.

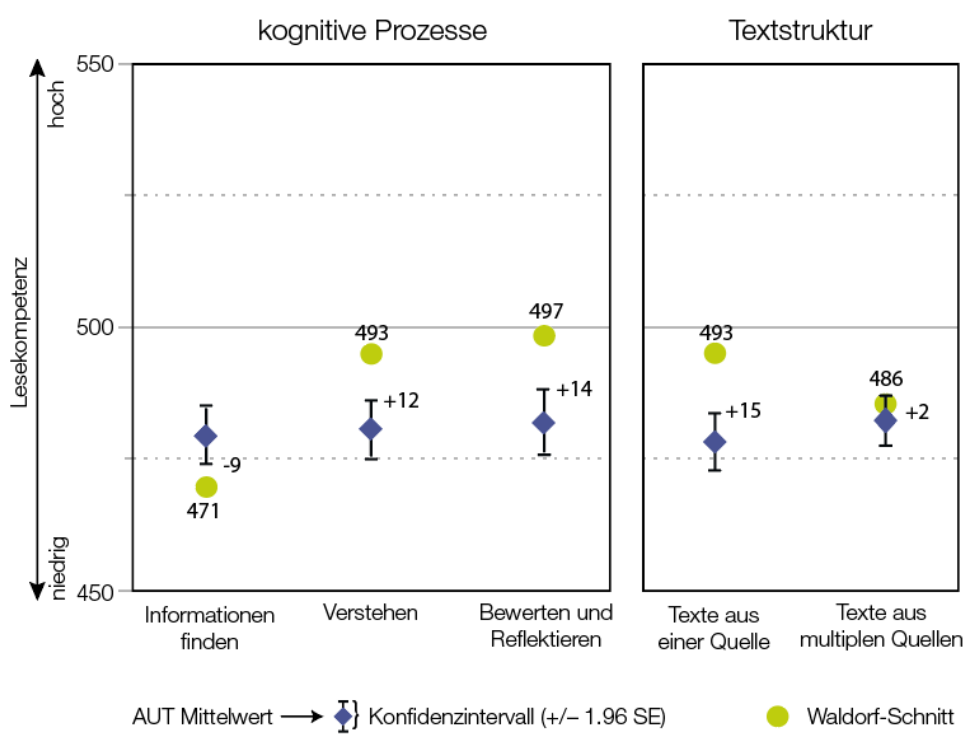

Abbildung 5: Mittelwertvergleiche in den Lesesubskalen - Waldorf- und Regelschulen im Vergleich (PISA 2018).

\subsection{Mathematikkompetenz}

In Mathematik können die Ergebnisse für PISA 2003 bis 2012 sowie für 2015 bis 2018 gegenübergestellt werden. Abbildung 6 zeigt die durchschnittliche Mathematikkompetenz der Schüler*innen in Waldorfschulen im Vergleich zu jenen in den österreichischen Regelschulen im Längsschnitt.

Während die Jugendlichen der Regelschulen in Mathematik von 2003 bis 2012 (mit Ausnahme von 2009) ähnlich hohe Mittelwerte (505 bzw. 506 Punkte) erreichen, zeigt sich für die Jugendlichen der Waldorfschulen von 2003 bis 2009 ein leichter Abwärtstrend um 10 bzw. 8 Punkte. 2012 ist die Mathematikleistung der Waldorfschüler*innen (495 Punkte) ähnlich dem Ergebnis von 2009 (492 Punkte). Mit diesen Ergebnissen 
R\&E-SOURCE $h$ ttps://journal.ph-noe.ac.at Online Journal for Research and Education

Ausgabe 16, Oktober 2021, ISSN: 2313-1640

unterscheiden sich die Waldorfschüler*innen bei PISA 2003 bis 2009 nicht signifikant von den Jugendlichen der Regelschulen. 2012 sowie 2015 liegen die Waldorfschüler*innen mit einer Differenz von 11 bzw. 8 Punkten signifikant unter dem Gesamtmittelwert von Österreich. 2018 erreichen die Waldorfschüler*innen mit 497 Testpunkten einen ähnlich hohen Mittelwert in Mathematik wie die Jugendlichen in Regelschulen.

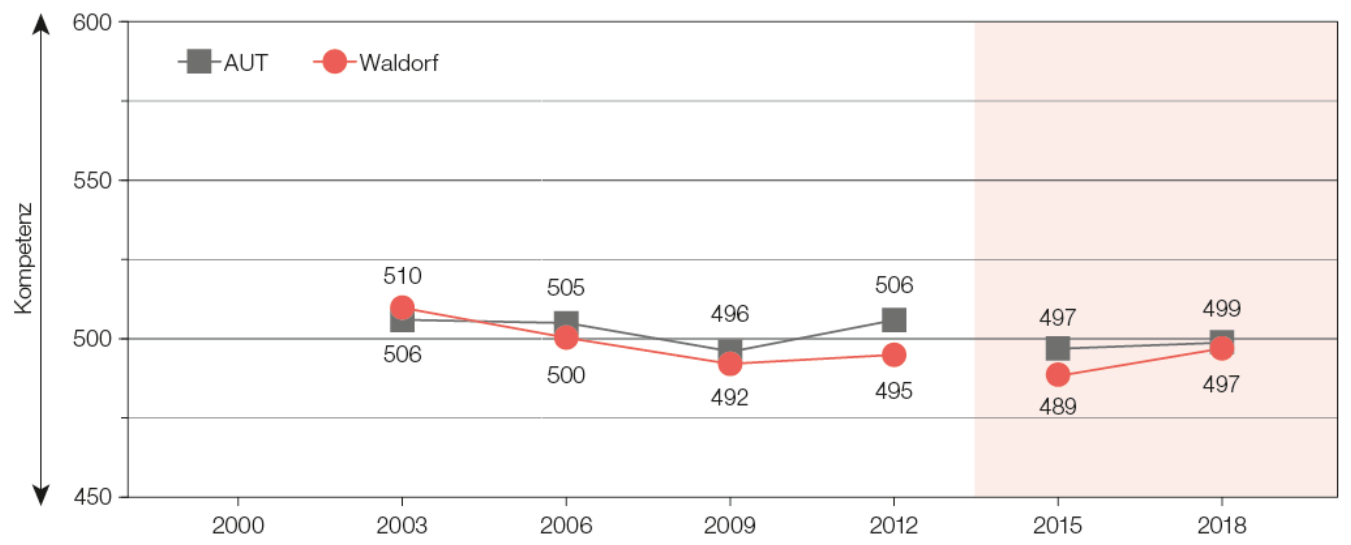

Abbildung 6: Veränderung der Durchschnittsleistung in Mathematik - Waldorf- und Regelschulen im Vergleich (PISA 2003, 2006, 2009, 2012, 2015, 2018).

Abbildung 7 stellt die Mathematikkompetenz der Waldorf- und Regelschüler*innen für 2018 verteilt auf die Kompetenzstufen dar. Die Mathematikkompetenz wird seit PISA 2003 mit sechs aufsteigenden Kompetenzstufen beschrieben. Schüler*innen, die eine der beiden höchsten Kompetenzstufen 5 oder 6 erreichen, werden zur Spitzengruppe zusammengefasst. Alle Schüler*innen, deren Leistungen auf Kompetenzstufe 1 oder darunter liegen, werden zur Risikogruppe zusammengefasst (im Detail siehe Suchań \& Höller, 2019, S. 32).

Auf den beiden unteren Leistungslevels (Level 1 und unter 1) befinden sich $13 \%$ der Waldorfschüler*innen. Den Leistungslevels 5 und 6 sind $5 \%$ der Waldorfschüler*innen zugeordnet. Im Vergleich zur Gesamtstichprobe der österreichischen Regelschulen weisen die Waldorfschulen eine um 8 Prozentpunkte kleinere Risikogruppe sowie auch Spitzengruppe in Mathematik auf. Sowohl die Risiko- als auch die Spitzengruppe in Mathematik ist in den Waldorfschulen damit signifikant kleiner als in den österreichischen Regelschulen (Österreich gesamt).

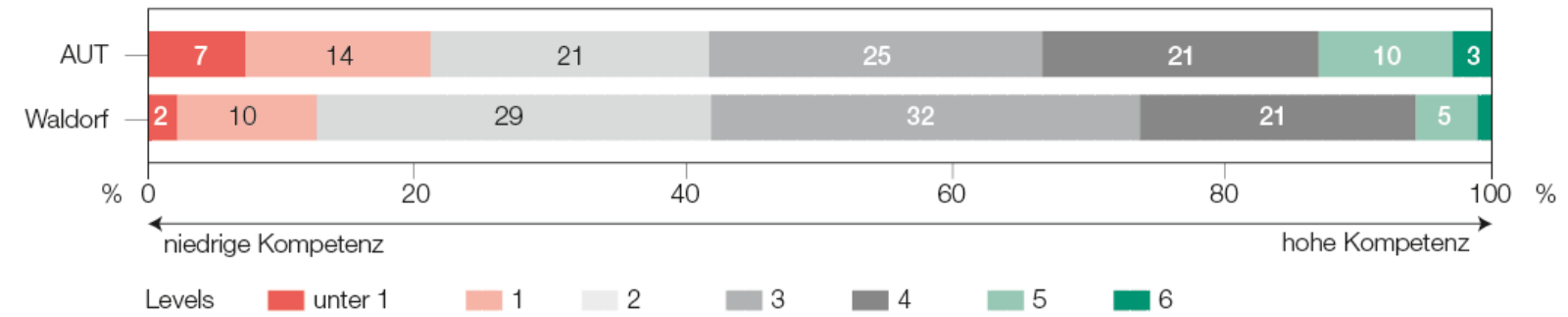

Abbildung 7: Kompetenzstufen in Mathematik - Waldorf- und Regelschulen im Vergleich (PISA 2018).

\subsection{Naturwissenschaftskompetenz}

Da die Naturwissenschaftskompetenz bei PISA 2006 erstmals die Hauptdomäne darstellt, sind Längsschnittvergleiche erst ab diesem Zyklus möglich. Abbildung 8 zeigt die durchschnittliche Naturwissenschaftskompetenz der Waldorfschüler*innen im Vergleich zu jener der Schüler*innen der österreichischen Regelschulen für PISA 2006 bis 2012 sowie 2015 bis 2018. Die Waldorfschüler*innen haben bei PISA 2006 und 2009 mit einem Leistungsmittelwert von 524 bzw. 523 ungefähr dieselbe durchschnittliche Leistung in Naturwissenschaft erzielt und sich 2012 um 11 bzw. 12 Punkte verschlechtert. Von 2015 auf 2018 zeichnet sich mit 3 Leistungspunkten Unterschied ein marginaler Abwärtstrend ab. Mit diesen Ergebnissen liegen die Waldorfschüler*innen bei PISA 2006 bis 2015 signifikant über dem Gesamtmittelwert von Österreich. 2018 erzielen die Jugendlichen in Waldorfschulen im Schnitt 500 Testpunkte und liegen mit 10 Punkten signifikant über dem Mittelwert der österreichischen Regelschulen. Der größte Unterschied zu den österreichischen 
Regelschulen zeigt sich bei PISA 2009 mit einer Differenz von 29 Testpunkten. Zu diesem Erhebungszeitpunkt lag die österreichische Naturwissenschaftsleistung im Gegensatz zu den anderen Erhebungszeitpunkten signifikant unter dem OECD-Schnitt (501 Punkte).

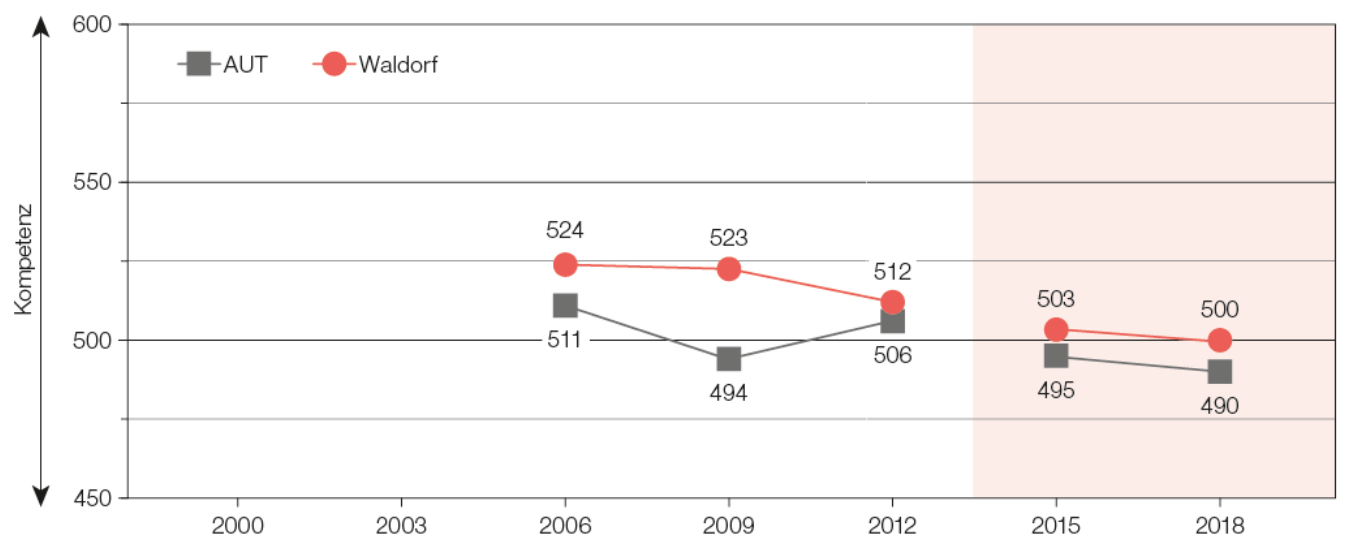

Abbildung 8: Veränderung der Durchschnittsleistung in Naturwissenschaft - Waldorf- und Regelschulen im Vergleich (PISA 2006, 2009, 2012, 2015, 2018).

Abbildung 9 stellt die Naturwissenschaftskompetenz der Waldorf- und Regelschüler*innen für 2018 verteilt auf die Kompetenzstufen dar. Die Naturwissenschaftskompetenz wird seit PISA 2006 mit sechs aufsteigenden Kompetenzstufen beschrieben. Schüler*innen, die eine der beiden höchsten Kompetenzstufen, 5 oder 6 , erreichen, werden zur Spitzengruppe zusammengefasst. Als Risikogruppe gelten Schüler*innen auf den Kompetenzstufen 1a, 1b und darunter (im Detail siehe Suchań \& Höller, 2019, S. 32).

In Naturwissenschaft zeigt sich für Österreich mit $22 \%$ wieder ein ähnlich hoher Risikoanteil wie bereits in Lesen und Mathematik. In den Waldorfschulen ist der Risikoanteil mit $12 \%$ wieder deutlich geringer. Der Spitzenanteil ist mit $6 \%$ in Österreich und $2 \%$ in den Waldorfschulen ähnlich groß wie im Kompetenzbereich Lesen.

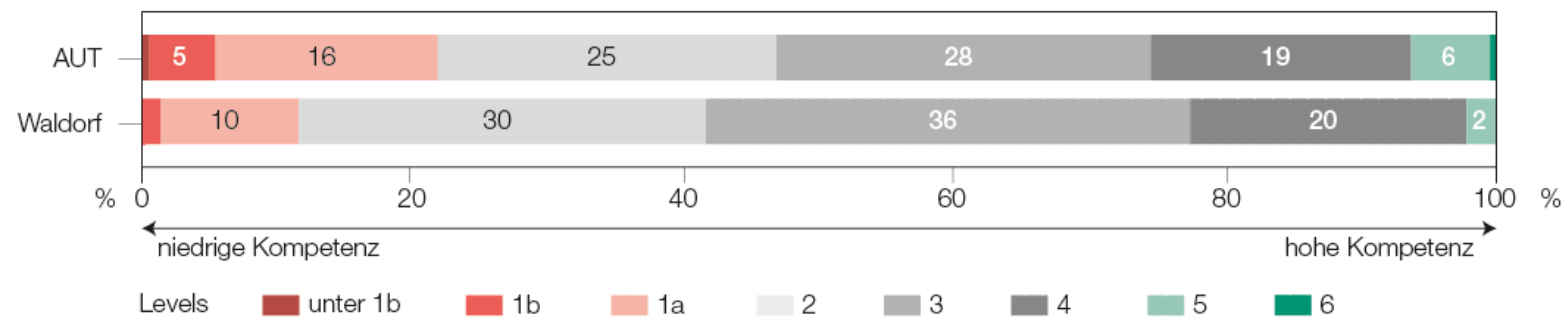

Abbildung 9: Kompetenzstufen in Naturwissenschaft - Waldorf- und Regelschulen im Vergleich (PISA 2018).

\subsection{Kompetenzen und Geschlechterunterschiede}

Abbildung 10 zeigt die Geschlechterdifferenzen der Waldorfschulen (Kreis) im Vergleich zu den österreichischen Regelschulen (Balken) für alle drei Kompetenzbereiche bei PISA 2018. Oberhalb der Null-Linie liegen die Balken, wenn die Burschen bessere Leistungen erbringen; unterhalb dieser Linie liegen die Balken, wenn die Mädchen höhere Durchschnittswerte erzielen.

Die Leistungsunterschiede zwischen Mädchen und Burschen stellen sich für Österreich gesamt in den drei Kompetenzbereichen wie folgt dar: In Naturwissenschaft unterscheiden sich Mädchen und Burschen nicht hinsichtlich ihrer Leistung. In Mathematik ist der Vorsprung der Burschen signifikant größer. Hier übertreffen Burschen die Mädchen im Schnitt um 13 Punkte. Im Lesen übertreffen hingegen die Mädchen ihre Mitschüler und schneiden um 28 Punkte besser ab. Die Unterschiede zwischen Mädchen und Burschen in den Waldorfschulen sind ähnlich hoch wie in den Regelschulen. Das heißt, das Waldorf- und Regelschulen sich nicht 
signifikant vom Österreich-Schnitt unterscheiden und bei den Geschlechterunterschieden ein vergleichbares Muster aufweisen.

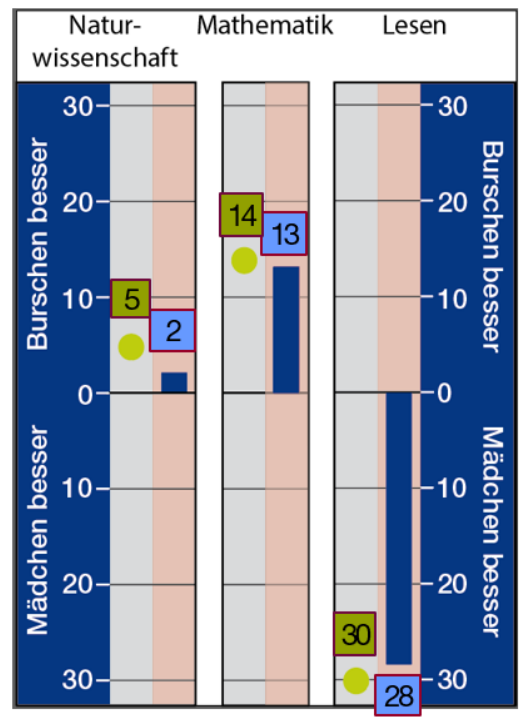

I AUT gesamt

Waldorfschulen

Abbildung 10: Unterschiede zwischen Mädchen und Burschen - Waldorf- und Regelschulen im Vergleich (PISA 2018).

\section{Motivation und Selbstwahrnehmung in Lesen}

Erfolgreiches Lernen und folglich auch der Erwerb bestimmter Kompetenzen werden von unterschiedlichen Faktoren beeinflusst. Gerade der sich von der Regelschule unterscheidende pädagogische und didaktische Zugang der Waldorfschulen lässt Unterschiede bei motivationalen Faktoren und selbstbezogenen Kognitionen der Waldorfschüler*innen vermuten. In diesem Abschnitt werden (1) die Lesefreude und (2) das Leseselbstkonzept der Waldorfschüler*innen im Vergleich zum OECD- und Österreich-Schnitt sowie zu den österreichischen Schulsparten betrachtet.

Die hier dargestellten Konstrukte stammen aus dem Schülerfragebogen zu PISA 2018 und beruhen auf den kumulierten Antworten auf jeweils mehrere einzelne Statements (Details zu diesen Konstrukten finden sich bei Höller, Lindemann, Wallner-Paschon \& Schaubmair, 2019, S. 66-71). Die Werte sind z-standardisiert, d. h., der OECD-Mittelwert ist 0 und die Standardabweichung 1 . Werte im positiven Bereich liegen über dem OECD-Schnitt und zeigen eine höhere Ausprägung des Konstrukts an. Negative Werte (unter dem OECD-Schnitt) kennzeichnen eine geringere Ausprägung.

Die Lesefreude der Jugendlichen wurde mit Hilfe von fünf Zustimmungsfragen wie beispielsweise „Lesen ist eines meiner liebsten Hobbys" erhoben. Abbildung 11 zeigt die durchschnittliche Lesefreude der Waldorfschüler*innen im Vergleich zu den österreichischen Schulsparten des Regelsystems sowie zum Österreich-Schnitt. Innerhalb der Schulsparten ist die Lesefreude der Jugendlichen in den AHS am höchsten und in den Berufsschulen (BS) mit Abstand am geringsten. Es ist ersichtlich, dass die Lesefreude der Waldorfschüler*innen - gekennzeichnet durch eine grüne Linie - weit über dem Österreich-Mittelwert liegt. Auch im Vergleich zu den Schulsparten im österreichischen Regelsystem sowie dem OECD-Schnitt weisen Waldorf-Schüler*innen ein jeweils stärker ausgeprägtes Maß an Lesefreude auf. 


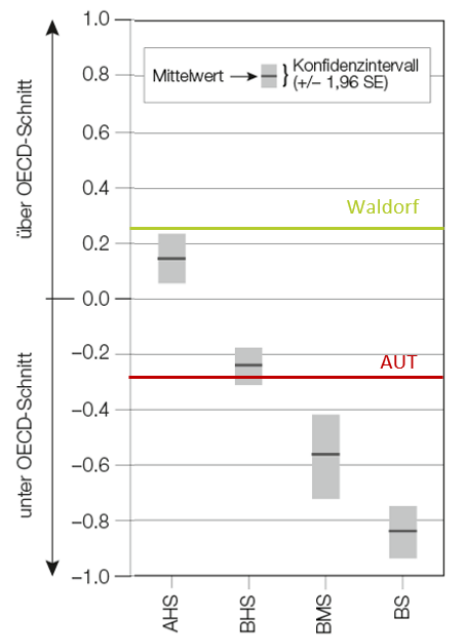

Abbildung 11: Lesefreude in Waldorfschulen im Vergleich (PISA 2018).

Beim Selbstkonzept geht es um die Wahrnehmung der eigenen Kompetenz. Bei PISA 2018 wird dabei zwischen der Wahrnehmung der eigenen Lesekompetenz sowie der Wahrnehmung von Schwierigkeiten beim Lesen unterschieden. Die Wahrnehmung der eigenen Lesekompetenz wurde mit 3 Zustimmungsfragen wie beispielsweise „Ich bin ein guter Leser/eine gute Leserin“ erhoben. Die Schüler*innen der Waldorfschulen haben eine signifikant positivere Wahrnehmung ihrer eigenen Lesefähigkeiten, als dies im OECD- und ÖsterreichDurchschnitt der Fall ist (s. Abbildung 12, linke Grafik). Betrachtet man die Schulsparten, zeigt sich, dass das Leseselbstkonzept der Waldorfschüler*innen mit jenem der AHS-Schüler*innen vergleichbar ist.

Die Wahrnehmung von Leseschwierigkeiten wurde ebenfalls mit 3 Zustimmungsfragen wie beispielsweise „Ich hatte schon immer Schwierigkeiten beim Lesen“ erhoben. Die Schülerantworten zu den einzelnen Aussagen wurden so zusammengefasst, dass eine höhere Wahrnehmung von Leseschwierigkeiten einem höheren Punktwert entspricht. Bei Betrachtung der Durchschnittswerte in Abbildung 12 (rechte Grafik) zeigt sich, dass in Österreich die Wahrnehmung von Leseschwierigkeiten gering ist und deutlich unter dem OECD-Schnitt liegt. Die Wahrnehmung von Schwierigkeiten beim Lesen bei den Jugendlichen in den AHS ist deutlich geringer als in den anderen Schulsparten. Die Jugendlichen in berufsbildenden mittleren Schulen (BMS) und Berufsschulen (BS) sind sich in der Einschätzung ihrer Leseschwierigkeiten sehr ähnlich und liegen signifikant über dem Durchschnittswert der berufsbildenden höheren Schulen (BHS).

Waldorfschüler*innen liegen mit der Wahrnehmung ihrer Leseschwierigkeiten im Österreich-Schnitt und damit ebenfalls unter dem OECD-Schnitt. Sie schätzen ihre Schwierigkeiten beim Lesen ähnlich hoch ein wie die Jugendlichen in den BHS.
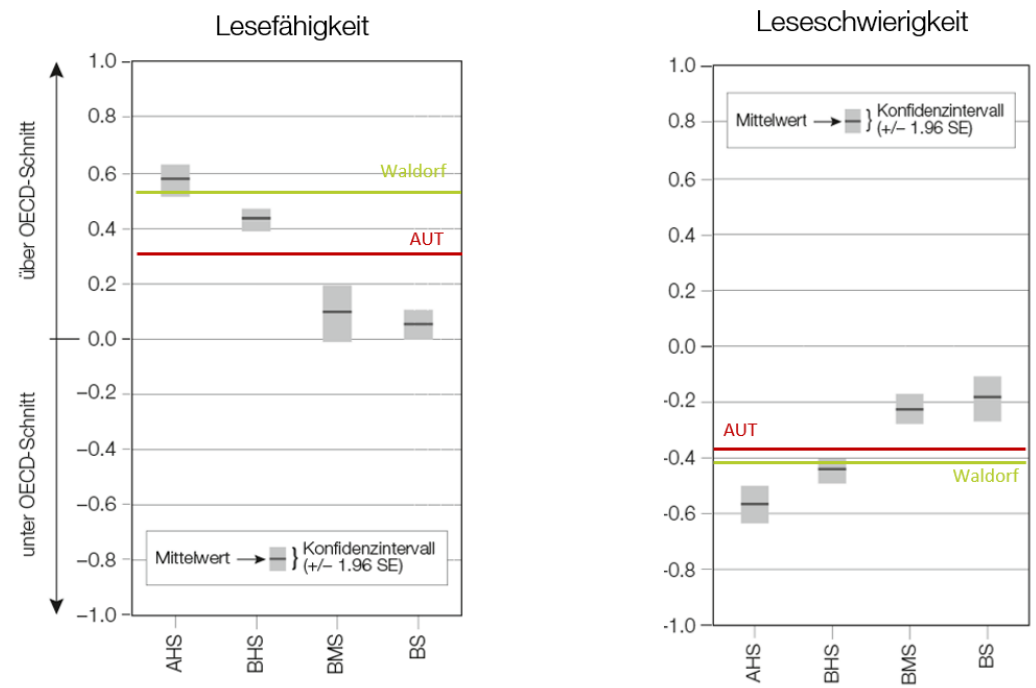

Abbildung 12: Wahrnehmung der Lesefähigkeit und Wahrnehmung von Leseschwierigkeiten in Waldorfschulen im Vergleich (PISA 2018). 


\section{Resümee}

Die in diesem Beitrag dargestellten Ergebnisse zu den kognitiven und nicht-kognitiven Kompetenzen der Waldorfschüler*innen aus PISA 2018 weisen keine Widersprüche zu den bisherigen Ergebnissen seit PISA 2000 auf. Die Kompetenzen der Waldorfschüler*innen in Mathematik, Naturwissenschaft und Lesen sind mit wenigen Ausnahmen über die Erhebungszeitpunkte hinweg relativ stabil.

Während die Waldorfschüler*innen bei PISA 2018 in Lesen und Naturwissenschaft moderat bessere durchschnittliche Leistungen erbringen als die Jugendlichen des österreichischen Regelschulwesens, erzielen sie in Mathematik ähnlich hohe Mittelwerte wie der Österreich-Schnitt. Waldorfschulen haben in allen drei Kompetenzbereichen kleinere Risiko- und Spitzengruppen als Regelschulen. Der Anteil an Jugendlichen mit mangelnden Grundkompetenzen beträgt in Mathematik und Naturwissenschaft jeweils $12 \%$ und in Lesen $16 \%$. Den größten Anteil an Risikoschüler*innen innerhalb der Waldorfschulen gibt es somit in Lesen. Spitzenleistungen in Mathematik zeigen $5 \%$ der Jugendlichen in Waldorfschulen, in Lesen $4 \%$ und in Naturwissenschaft $2 \%$.

Bei den Detailanalysen zur Lesekompetenz zeigt sich bei den Waldorfschüler*innen, im Gegensatz zu den Jugendlichen in Regelschulen, ein klares Kompetenzprofil. Ihre relativen Stärken liegen beim Verstehen von Texten und somit beim Wiedergeben, Vernetzen und Schlussfolgern aus Textinhalten. Aber auch beim Bewerten und Reflektieren von Texten sowie beim Bearbeiten von Texten aus einer Quelle zeigen sich ihre Stärken.

Hinsichtlich der Geschlechterdifferenzen unterscheiden sich die 15-/16-Jährigen in Waldorfschulen nicht wesentlich vom Österreich-Schnitt. Während die Burschen in Naturwissenschaft und Mathematik bessere Leistungen erbringen, übertreffen die Mädchen in Lesen ihre Mitschüler.

Bezüglich der motivationalen Merkmale der Schüler*innen zeigt sich in den Waldorfschulen im Einklang mit bisherigen Untersuchungen (Reiter, 2002; Wallner-Paschon, 2006, 2009, 2018) ein positives Bild. Die Lesefreude der Waldorfschüler*innen liegt deutlich über den Mittelwerten der österreichischen Schulsparten und dem Österreich-Schnitt. Auch das Leseselbstkonzept lässt sich als positiv charakterisieren. Dabei zeigt sich, dass Jugendliche in Waldorfschulen ihre Lesekompetenz positiver wahrnehmen als im OECD- und ÖsterreichDurchschnitt. Mit ihrer Wahrnehmung von Leseschwierigkeiten liegen sie im Österreich-Schnitt und unter dem OECD-Durchschnittswert. Ähnlich positive Ergebnisse berichten Liebenwein, Barz \& Randoll (2012, S. 59 ff.) auch für die allgemeine Lernfreude, das Lerninteresse sowie die allgemeine schulische Selbstwirksamkeitserwartung der Waldorfschüler*innen.

PISA misst die Kompetenzen der Schüler*innen im Alter von 15 bis 16 Jahren. In diesem Alter haben sie in der Regel 9 Jahre die Schule besucht und befinden sich am Ende ihrer Pflichtschulzeit. PISA bietet somit einen idealen Zeitpunkt, um den schulischen Output zwischen zwei Schulformen zu vergleichen. Dennoch müssen der Vergleich und das Abschneiden der Waldorfschüler*innen mit dem Wissen um die andere Zusammensetzung der Population mit Vorsicht interpretiert werden. Schüler*innen in Waldorfschulen haben häufiger Eltern mit einem höheren Berufsstatus sowie höheren Bildungsabschlüssen. Hinsichtlich dieser Merkmale sind sie nicht mit der Gesamtheit der österreichischen Schülerpopulation vergleichbar, sondern am ehesten mit Schüler*innen, die eine AHS besuchen (Wallner-Paschon, 2009, S. 389).

\section{Literatur}

Barz, H., Randoll, D. (Hrsg.) (2007). Absolventen von Waldorfschulen. Eine empirische Studie zu Bildung und Lebensgestaltung. 2. Auflage. Wiesbaden: Springer VS.

Ganzeboom, H. B. G., De Graaf, P. M. \& Treiman, D. J. (1992). A standard international socio-economic index of occupational status. Social Science Research, 21, 1-56. doi: http://dx.doi.org/10.1016/0049089X(92)90017-B.

Gläser, A. \& Pareiss, M. (2019). Rücklauf, Stichprobenausfälle und Stichprobengrößen bei PISA 2018. In B. Suchań (Hrsg.). PISA 2018. Technischer Bericht (S. 65-78). Salzburg: IQS

Hellmich, A. (1999). Rudolf Steiner. In A. Hellmich \& P. Teigeler (Hrsg.), Montessori-, Freinet-, Waldorfpädagogik. Konzeption und aktuelle Praxis (S. 50-57). Weinheim, Basel: Beltz.

Höller, I., Lindemann, R., Wallner-Paschon, C. \& Schaubmair, V. (2019). Kompetenzentwicklung im Kontext individueller und familiärer Faktoren. In B. Suchań, I. Höller \& C. Wallner-Paschon (Hrsg.), PISA 2018. Grundkompetenzen am Ende der Pflichtschulzeit im internationalen Vergleich (S. 66-80). Graz: Leykam. http://doi.org/10.17888/pisa2018-eb 
Höller, I. \& Toferer, B. (2019). Die getesteten Kompetenzbereiche. In B. Suchań (Hrsg.). PISA 2018. Technischer Bericht. (S. 13-24). Salzburg: IQS

Liebenwein, S., Barz, H. \& Randoll, D. (2012). Bildungserfahrungen an Waldorfschulen. Empirische Studie zu Schulqualität und Lernerfahrungen. Wiesbaden: Springer.

Loebell, P. (2018). Waldorfpädagogik. In B. Heiner (Hrsg.), Handbuch Bildungsreform und Reformpädagogik (S. 245-259). Springer VS, Wiesbaden.

Pareiss, M. (2019). Sampling-Design und Stichproben. In B. Suchań (Hrsg.). PISA 2018. Technischer Bericht (S. 35-48). Salzburg: IQS

Rauthe, W. (1992). Erfahrungen mit dem Epochenunterricht in der Waldorfschule. In S. Leber (Hrsg.). Die Pädagogik der Waldorfschule und ihre Grundlagen (S. 285-293). Darmstadt: Wissenschaftliche Buchgesellschaft.

Salchegger, S., Wallner-Paschon, C. \& Bertsch, C. (2021). Explaining Waldorf students' high motivation but moderate achievement in science: is inquiry-based science education the key? Large-scale Assess Educ 9, 14 https://doi.org/10.1186/s40536-021-00107-3

Randoll, D. (2004). Die Freie Waldorfschule zwischen Anspruch und Wirklichkeit. Eine vergleichende Untersuchung zu Schülerurteilen aus Waldorfschulen und Gymnasien. In H. Ullrich, T. Idel \& K. Kunze (Hrsg.). Das Andere Erforschen. Empirische Impulse aus Reform und Alternativschulen (2. Aufl.). (S. 3550). Wiesbaden: Verlag für Sozialwissenschaften.

Reiter, C. (2002). Ein Leistungsprofil der österreichischen Waldorfschulen. In C. Wallner-Paschon (Hrsg.). PISA Plus 2000. Thematische Analysen nationaler Projekte. Innsbruck: Studien Verlag.

Suchań, B. \& Breit, S. (2016). PISA 2015 - eine Einführung in die aktuelle Studie. In B. Suchań \& S. Breit (Hrsg.). PISA 2015. Grundkompetenzen am Ende der Pflichtschulzeit im internationalen Vergleich (S. 9-37). Graz: Leykam.

Suchań, B. \& Höller, I. (2019). PISA 2018 - eine Einführung in die aktuelle Studie. In B. Suchań, I. Höller \& C. Wallner-Paschon (Hrsg.). PISA 2018. Grundkompetenzen am Ende der Pflichtschulzeit im internationalen Vergleich. (S. 9-38). Graz: Leykam

Ullrich, H. (2004). Forschung über Waldorfschulen - auf neuen Wegen. In H. Ullrich, T. Idel \& K. Kunze (Hrsg.). Das Andere Erforschen. Empirische Impulse aus Reform und Alternativschulen (S. 21-34). Wiesbaden: Verlag für Sozialwissenschaften.

Verband der Waldorfschulen (2005). Die Freie Waldorfschule und das Konzept der Ganztagsschule. In S. Appel, H. Ludwig U. Rother \& G. Rut (Hrsg.). Schulkooperationen (S. 253-256). Schwalbach, Taunus: Wochenschau.

Wallner-Paschon, C. (2018). Kompetenzen, Motivation und Selbstwahrnehmung der Waldorfschüler/innen im Vergleich. Journal for Research and Education, 9. Verfügbar unter: https://journal.phnoe.ac.at/index.php/resource/article/view/548/543

Wallner-Paschon, C. (2009). Kompetenzen und individuelle Merkmale der Waldorfschüler/innen im Vergleich. In C. Schreiner \& U. Schwantner (Hrsg.). PISA 2006. Österreichischer Expertenbericht zum Naturwissenschafts-Schwerpunkt (S. 387-399). Graz: Leykam. Verfügbar unter http://www.bifie.at/wpcontent/uploads/2017/05/PISA2006 NEB web.pdf, Zugriff am 01.01.2018.

Wallner-Paschon, C. (2006). Kompetenzen und individuelle Merkmale der Waldorfschüler/innen. In G. Haider \& C. Schreiner (Hrsg.). Die PISA-Studie. Österreichs Schulsystem im internationalen Wettbewerb (S. 143154). Wien: Böhlau. 